Pacific Journal of Mathematics

AN ALMOST CLASSIFICATION OF COMPACT LIE GROUPS 


\title{
AN ALMOST CLASSIFICATION OF COMPACT LIE GROUPS WITH BORSUK-ULAM PROPERTIES
}

\section{WaClaw Marzantowicz}

\begin{abstract}
We say that a compact Lie group $G$ has the Borsuk-Ulam property in the weak sense if for every orthogonal representation $V$ of $G$ and every $G$-equivariant map $f: S(V) \rightarrow S(V), V^{G}=\{0\}$, of the unit sphere we have $\operatorname{deg} f \neq 0$.

We say that $G$ has the Borsuk-Ulam property in the strong sense if for any two orthogonal representations $V, W$ of $G$ with $\operatorname{dim} W=$ $\operatorname{dim} V$ and $W^{G}=V^{G}=\{0\}$ and every $G$-equivariant map $f: S(V)$ $\rightarrow S(W)$ of the unit spheres we have $\operatorname{deg} f \neq 0$. In this paper a complete classification, up to isomorphism, of group with the weak Borsuk-Ulam property is given. A classification of groups with the strong Borsuk-Ulam property does not cover nonabelian $p$-groups with all elements of the order $p$. In fact we deal with a more general definition admitting a nonempty fixed point set of $G$ on the sphere $S(V)$.
\end{abstract}

1. The main theorems. In order to formulate our main results we introduce the following notation. Let $G$ be a compact Lie group. We denote by $G_{0}$ the component of identity of and by $\Gamma$ the quotient group $G / G_{0}$. We use standard notation of the theory of compact transformation groups (see for instance [4] or [5]). In particular, for every subgroup $H \subset G$, the fixed point set of $H$ on a $G$-space $X$ is denoted by $X^{H}$. Also, for a $G$-equivariant map $f: X \rightarrow Y$ between two $G$-spaces, we denote by $f^{H}$ its restriction to the space $X^{H}$. The symbol $(n, m)$ stands for the greatest common divisor of the integers $n, m$ with the notation $(0,1)=0$ and $|G|$ for the rank of the (finite) group $G$. We will work with the following definition of the BorsukUlam property (cf. [11]).

Definition I. (A) We say that $G$ has the Borsuk-Ulam property in the weak sense $A$ if for every orthogonal representation $V$ of $G$ and every $G$-equivariant map $f: S(V) \rightarrow S(V)$ if $\left(\operatorname{deg} f^{G},|\Gamma|\right)=1$ then

$$
\operatorname{deg} f \neq 0 \text {. }
$$

REMARK. Note that in the case $V^{G}=\{0\}$ there is no condition on $\operatorname{deg} f^{G}$ and the property then requires that $\operatorname{deg} f \neq 0$ for every $G$ equivariant map. Also, if $G=G_{0}$ then the condition $\left(\operatorname{deg} f^{G},|\Gamma|\right)=$ 1 means that $\operatorname{deg} f^{G} \neq 0$. 
(B) We say that $G$ has the Borsuk-Ulam property in the weak sense $B$ if for every pair or orthogonal representations $W \subsetneq V$ of $G$, with $V^{G}=\{0\}$, there is no $G$-equivariant map $f: S(V) \rightarrow S(W)$.

Definition II. (A) We say that $G$ has the Borsuk-Ulam property in the strong sense $A$ if for every pair of orthogonal representations $V, W$ of $G$, with $\operatorname{dim} W=\operatorname{dim} V, \operatorname{dim} V^{G}=\operatorname{dim} V^{G}$, and every $G$-equivariant map

$$
f: S(V) \rightarrow S(W)
$$

if $\left(\operatorname{deg} f^{G},|\Gamma|\right)=1$ then $\operatorname{deg} f \neq 0$. (If $W^{G}=\{0\}$ or $G=G_{0}$, the same remark as above applies.)

(B) We say that $G$ has the Borsuk-Ulam property in the strong sense $B$ if there is no $G$-equivariant map $f: S(V) \rightarrow S(W)$ where $W$ is an orthogonal representation of $G$ such that $W \subsetneq U$ for an orthogonal representation $U, \operatorname{dim} U=\operatorname{dim} V$ and $U^{G}=\{0\}$.

To shorten notation, we say that $G$ has property I.A (respectively I.B, II.A, and II.B) if $G$ has the Borsuk-Ulam property in the sense of Definition I.A (respectively Def. I.B, Def. II.A and Def. II.B).

We now state a simple observation we shall frequently use.

1.1. Proposition. With the above notation we have the following implications:

$$
\begin{array}{ccc}
\begin{array}{c}
\text { has II.A } \\
\Downarrow
\end{array} & G \text { has II.B } \\
\Downarrow \text { has I.A } & \Rightarrow & G \text { has I.B }
\end{array}
$$

Proof. Indeed, as in the classical $Z_{2}$-case, composing $f$ with the inclusion of $S(W)$ into $S(U)$ (or into $S(V)$ ) we get a $G$-equivariant map from $S(V)$ into $S(V)$ (or into $S(U)$ ) of degree 0, which gives the horizontal implications. The vertical implications are evident.

We shall use the symbol $T^{k}$ to denote the $k$-dimensional torus $S^{1} \times \cdots \times S^{1}$ and $Z_{p}^{r}$ for the $p$-torus $Z_{p} \times \cdots \times Z_{p}, p$-prime.

Our main result formulates as follows.

THEOREM 1. A compact Lie group $G$ has the Borsuk-Ulam property I.A. if and only if it is an extension

$$
T^{k} \rightarrow G \rightarrow \Gamma
$$

of the $k$-dimensional torus, $k \geq 0$, by a p-group $\Gamma$, where $p$ is a prime or $q$. 
THEOREM 2. (a) If a compact Lie group $G$ has the Borsuk-Ulam property II.A, then it is an extension

$$
T^{k} \rightarrow G \rightarrow \Gamma
$$

of $T^{k}, k \geq 0$, by a p-group $\Gamma$ such that every element of $\Gamma$ has the order $p$, p-prime or 1 .

(b) The product $G=T^{k} \times Z_{p}^{r}$ of the torus $T^{k}, k \geq 0$, by the $p$-torus $Z_{p}^{r}, r \geq 0$, has the Borsuk-Ulam property II.A.

In a first step we will show that it is sufficient to study a connected compact Lie group and a nontrivial finite group only.

1.2. Lemma. Let $G_{0} \rightarrow G \rightarrow \Gamma$ be the canonical extension of a compact Lie group $G$. Then if $G_{0}$ has I.A. (resp. II.A) and $\Gamma$ has I.A. (resp. II.A) then $G$ has this property too.

Proof. Since $G_{0}$ is the normal subgroup, $\Gamma=G / G_{0}$ acts on $V^{G_{0}}$ and $V^{G}=\left(V^{G_{0}}\right)^{\Gamma}$. Moreover; for every $G$-map $f: S(V) \rightarrow S(V)$ its restriction $\left.f\right|_{V^{G_{0}}}: S\left(V^{G_{0}}\right) \rightarrow S\left(V^{G_{0}}\right)$ is a $\Gamma$-equivariant map. Suppose that $\left(\operatorname{deg} f^{G},|\Gamma|\right)=1$ or $V^{G}=\{0\}$, then from property I.A. of $\Gamma$ it follows that $\operatorname{deg} f^{G_{0}} \neq 0$, and consequently $\operatorname{deg} f \neq 0$ by property I.A. of $G_{0}$. The proof for property II.A. is similar.

1.3. Lemma. Suppose that there exists an orthogonal representation $V$ of $G_{0}$ with $V^{G_{0}}=\{0\}$ and a $G_{0}$-map $f: S(V) \rightarrow S(V)$ of degree 0 , or that $\Gamma$ fails to have property I.A. Then $G$ fails to have property I.A, and consequently II.A.

Proof. Suppose first, contrary to our claim, that for a group $G$ with property I.A., there exist an orthogonal representation of $\Gamma$ with $V^{\Gamma}=\{0\}$ and a $\Gamma$-equivariant map $f: S(V) \rightarrow S(V), \operatorname{deg} f^{\Gamma} \neq 0$, $\left(\operatorname{deg} f^{\Gamma},|\Gamma|\right)=1$ and $\operatorname{deg} f=0$. Note that the natural projection $G \rightarrow \Gamma$ makes $V$ an orthogonal representation of $G$ with $V^{G}=V^{\Gamma}$. Moreover the map $f: S(V) \rightarrow S(V)$ is $G$-equivariant with respect to this action of $G$, which contradicts property I.A. of $G$.

In order to prove the lemma under the first part of assumption we have to use the induced representation from a subgroup. This fact was explored in the work of M. Atiyah and D. Tall ([1], II §2. Prop. 2.2).

1.4. Let $V, W$ be orthogonal representation of a compact Lie group $H$ and $f: S(V) \rightarrow S(W)$ an $H$-map of degree $k$. If $H$ is a subgroup 
of a group $G$ with the finite quotient space $|G / H|=m$, then $f$ induces a $G$-equivariant map

$$
\operatorname{ind}_{*} f: S(\text { ind } V) \rightarrow S(\text { ind } W)
$$

of the spheres of the induced representations of $G$, which is of degree $k^{m}$.

As a matter of fact, in [1] the above statement was proved only for the case of unitary representations of a finite group $H$. Nevertheless, the same proof remains valid in the orthogonal case.

To construct a $G$-map contradicting property I.A. of $G$, it is sufficient to use (1.4) for $H=G_{0}$ together with the observation that (ind $V)^{G}=V^{0}=\{0\}$. Indeed, the degree of the $G$-equivariant map

$$
\operatorname{ind}_{*} f: S(\text { ind } V) \rightarrow S(\text { ind } W)
$$

is $(\operatorname{deg} f)^{|\Gamma|}=0$. This ends the proof of the lemma.

We can state now the following fact.

1.5. Proposition. An infinite compact Lie group $G$ has property I.A. and II.A. if and only if its component of the identity $G_{0}$ is isomorphic to the torus $T^{k}, k \geq 1$.

Proof. In 1966 W. C. Hsiang and W. Y. Hsiang ([7]) proved that for every connected nonabelian compact Lie group $G$ there exist an orthogonal representation $V$ of $G$ with $V^{G}=\{0\}$ and a $G$-equivariant map $f: S(V) \rightarrow S(V)$ such that $\operatorname{deg} f=0$. From this and Lemma 1.3 it follows that $G_{0}$ must be abelian.

On the other hand, in [11] the author proved that the torus $T^{k}, k \geq$ 1 , has property II.A, which completes the proof in respect of Proposition 1.1.

1.6. Proposition. Every finite p-group $\Gamma$, p-prime, has the BorsukUlam property I.A.

Proof. The statement follows from the results of $\mathrm{T}$. tom Dieck (see [5] for the complete bibliography of Dieck's result on this subject) or from author's results of [9] partially published in [10]. Indeed, the mentioned result says that for a $G$-equivariant map $f$ of a $G$-CW complex $X(S(V)$ is a $G$-CW complex being a smooth $G$-manifold [5]) we have the congruence of the Lefschetz numbers

$$
L(f) \cong L\left(f^{G}\right) \quad(\bmod p)
$$


if $G$ is a $p$-group. For a $G$-map of the sphere this gives the statement. In the author's work [11] it is shown that

\subsection{Proposition. The p-torus $Z_{p}^{r}, r \geq 1$, p-prime has property II.A.}

Later we shall show that if a $p$-group contains an element of the order $p^{2}$ then it fails to have property II.A.

Summing up the above, we are left with the task to show that if a finite group is not $p$-group then it fails to have property I.A. and if a $p$-group contains an element of the order $p^{2}$ then it fails to have property II.A.

1.8. Historical remarks. In our terminology, the classical BorsukUlam theorem states that $G=Z_{2}$ has property I.A., hence also I.B, if $V^{G}=\{0\} \quad([2])$. Observe that since $Z_{2}$ has only one nontrivial irreducible representation, property I.B. coincides with property II.B, and I.A. with II.A. It is known and not difficult to check that $Z_{2}$ has property I.A.

The case $G=Z_{p}, p$-prime, was studied by many authors and it is known as the Smith theorem. For example, in [8] it is shown that $\operatorname{def} f \cong \operatorname{deg} f^{G}(\bmod p)$ for every $G$-equivariant map $f: S(V) \rightarrow$ $S(W)$ as in Definition II.A. It follows that $Z_{p}$ has property II.A.

The case $G=S^{1}$ was extensively studied by several authors with a view to its applications to nonlinear analysis. In [10] it is shown that the torus $T^{k}=S^{1} \times \cdots \times S^{1}, k \geq 1$, has property I.A. Using analytic methods, L. Nirenberg proved that $G=S^{1}$ has property II.A. ([12]). This was also proved independently by E. Fadell, S. Husseini and P. Rabinowitz. They use the relative cohomological $S^{1}$-index introduced by them in [6]. It is worth pointing out that for $G=S^{1}$ the above result is covered by an earlier, unfortunately unpublished result of P. Traczyk. In 1977 he showed in his MSc. work that $S^{1}$ has property II.A. Moreover his simple geometrical method of proof gives also, as in [12], a relation between the characters of representations $V$ and $W$ for which a $G$-map $f: S(V) \rightarrow S(W)$ exists. This method is exposed in the work [11].

On the other hand, the mentioned Hsiangs result (compare with 1.5) states that every connected nonabelian compact Lie group does not have property I.A. Earlier, E. Floyd showed that for $Z_{p q}, p, q$ prime, $p \neq 1$, there exist a unitary representation $V$ of $G$ of complex dimension 2, with $V^{G}=\{0\}$, and a $G$-equivariant map 
$f: S(V) \rightarrow S(V)$ of degree 0 . The proof is presented in Bredon's book [4] ([4] I.8). This means that such a group fails to have property I.A.

2. Finite groups without the Borsuk-Ulam properties I.A. and II.A. We wish to show first that a group $G$ fails to have property I.A if its order has two distinct prime divisors, and secondly that a $p$-group $G$ containing the cyclic group $Z_{p^{2}}$ does not have property II.A.

The main idea of the proof is to find an example of an $H$-equivariant map of degree 0 , for some subgroups $H \subset G$, and next lift it to a $G$ equivariant map of the induced representation (1.4).

2.1. THEOREM. Let $G$ be a finite group such that two distinct prime numbers $p, q$ divide the order of $G$.

Then there exists an orthogonal representation $V$ of $G$ with $V^{G}=$ $\{0\}$ and a G-equivariant map $f: S(V) \rightarrow S(W)$ such that $\operatorname{deg} f=0$.

In the proof of Theorem 5, the basic geometric ingredient is the Conner-Floyd operation

$$
\text { 口: } \operatorname{Map}(X, X) \times \operatorname{Map}(Y, Y) \rightarrow \operatorname{Map}(X * Y, X * Y)
$$

(see [4] I.). It allows us to produce, for $|G|=p^{l} q, p, q$ primes, $p \neq q$, a $G$-equivariant map $f$ of the sphere a representation with $\operatorname{deg} f=0$. The proof falls naturally into two parts. We first examine the case of $|G|$ odd. We begin by proving the following lemma.

2.2. Lemma. Suppose that the statement of Theorem 2.1 holds for every group of order $p^{l} q$, where $p, q$ are distinct odd primes.

Then it also holds for every group $G$ of odd order such that at least two distinct primes divide the order of $G$.

Proof. We shall prove the lemma by induction on the order of $G$. Assume that, for a given $n \in N$, Theorem 5 holds for every group $G$ of odd order smaller than $n$ and let $G$ be a group of odd order $|G|=n$. By the Feit-Thompson theorem $G$ is solvable.

Let $G_{0}=\{e\} \subset G_{1} \subset \cdots \subset G_{m-1} \subset G_{m}=G$ be a resolution of $G$ with the factors being cyclic of prime order.

If $\left|G_{m-1}\right|=p^{l}$ for some prime $p$, then we are reduced to the case $|G|=p^{l} q$. Otherwise the statement of Theorem 2.1 holds for $G_{m-1}$ by the induction assumption. This means that there exist an orthogonal representation $V$ of $G_{m-1}$ with $V^{G_{m-1}}=\{0\}$ and a $G_{m-1}$ equivariant map $f: S(V) \rightarrow S(V)$ of degree zero. Using the induced representation ind $V$ of $G_{m}=G$ we construct a $G$-equivariant 
map $f: S($ ind $V) \rightarrow S($ ind $V$ ) of degree 0 (see 1.4), which proves the lemma.

We shall have established Theorem 2.1 for odd order groups if we prove the following proposition.

2.3. Proposition. Suppose that the order of $G$ is of the form $p^{l} q$, $p, q$ distinct odd primes, $l \geq 1$.

Then there exist an orthogonal representation $V$ of $G$ with $V^{G}=$ $\{0\}$ and a G-equivariant map $f: S(V) \rightarrow S(V)$ of degree 0 .

Proof. Observe first that we need only consider the case of $G$ being an extension of a $p$-group $H$ by $Z_{q}$. Indeed, let $G_{0}=\{e\} \subset$ $G_{1} \subset G_{2} \subset \cdots \subset G_{m-1} \subset G_{m}=G$ be a resolution of $G$ with cyclic factors. If we take $i_{0}$ to be the smallest number for which the numbers $\left|G_{i} / G_{i-1}\right|, i<i_{0}$, and $\left|G_{i_{0}} / G_{i_{0}-1}\right|$ are relatively prime, then we get a subgroup of $G$ of the desired form. Using once more the induced representation we get the conclusion for $G$. Recall, that for every $k \in Z$ there exist an orthogonal representation $V$ of $Z_{q}$ with $V^{Z_{q}}=\{0\}$ and a $Z_{q}$-equivariant map $f: S(V) \rightarrow S(V)$ such that $\operatorname{deg} f=k q+1$. Indeed, we can take $V$ to be the one-dimensional unitary representation $V^{1}$, and $f(z)=z^{k q-1}$. Since $G$ has an epimorphism $\varphi$ onto $Z_{q}, f$ can be considered as a $G$-equivariant map of $S(V)$ with respect to the $G$-structure given by $\varphi$. On the other hand, $H$ being $p$-group, it has an epimorphism $\psi$ onto $Z_{p}$.

Proceeding as above we can construct an orthogonal representation $\widetilde{W}$ of $H$ with $\widetilde{W}^{H}=\{0\}$ and an $H$-equivariant map $\tilde{h}: S(\widetilde{W}) \rightarrow$ $S(\widetilde{W})$ of degree $m p+1$, for every $m \in Z$. Taking the induced representation $W=$ ind $\widetilde{W}$, we get a $G$-equivariant map $h: S(W) \rightarrow$ $S(W)$ of degree $(m p+1)^{q}$ (see 1.4$)$.

We shall use the following well-known fact

2.4. For any orthogonal representation $V, W, V_{1}, W_{1}$ of $G$, with $\operatorname{dim} V_{1}=\operatorname{dim} V, \operatorname{dim} W_{1}=\operatorname{dim} W$, and $G$-equivariant maps $f: S(V)$ $\rightarrow S\left(V_{1}\right)$ and $h: S(W) \rightarrow S\left(W_{1}\right)$, the join $S(V) * S(W)$ is $G$-homeomorphic to $S(V \in W)$ and $\operatorname{deg}(f * h)=\operatorname{deg} f \cdot \operatorname{deg} h$.

With the maps constructed at the beginning of the proof, we can use the Conner-Floyd operation $\square$ ([4] II.8.3). We recall this construction for the convenience of the reader. For given CW-complexes $X$ and $Y$, there is a map

$$
\lambda: X * Y \rightarrow X * Y \cup_{Y} Y * X \cup_{X} X * Y
$$


defined by

$$
(t a,(1-t) b)= \begin{cases}(3 t a,(1-3 t) b) & \text { for } 0 \leq t \leq 1 / 3 \\ ((3 t-1) b,(2-3 t) a) & \text { for } 1 / 3 \leq t \leq 2 / 3 \\ ((3 t-2) a(3-3 t) b) & \text { for } 2 / 3 \leq t \leq 1\end{cases}
$$

If $f: X \rightarrow X$ and $h: Y \rightarrow Y$ are any maps, then one may form the union

$$
f * 1 \cup \tau \cup 1 * h: X * Y \cup_{Y} Y * Y \cup_{X} X * Y \rightarrow X * Y,
$$

where $\tau: Y * X \rightarrow X * Y$ is the canonical map $\tau(t b,(1-t) a)=$ $((1-t) a, t b)$. The operation $\square$ is defined by

$$
f \square h=(f * 1 \cup \tau \cup 1 * h) \cdot \lambda .
$$

If $X$ and $Y$ are spheres, then $\operatorname{deg}(f * 1)=\operatorname{deg} f$ and $\operatorname{deg}(1 * h)=\operatorname{deg} h$ (2.4) so that

$$
\operatorname{deg}(f \square h)=\operatorname{deg} f+\operatorname{deg} h-1 .
$$

Note that if $X$ and $Y$ are $G$-spaces and $f, h$ are $G$-equivariant then $f \square h$ is also equivariant, because all maps used in the definition of $\square$ are $G$-equivariant.

Adding $t$ times $f$ and $s$ times $h$ in the sense of the operation $\square$ we get

$$
\operatorname{deg}(\stackrel{t}{\underset{1}{L}} f \square \stackrel{s}{\square} h)=t(\operatorname{deg} f-1)+s(\operatorname{deg} h-1)+1 .
$$

Let us apply this to the $G$-equivariant maps $f$ and $h$ we have defined earlier. We get

$$
\operatorname{deg}\left(\underset{1}{t} f \square \stackrel{s}{L_{1}} h\right)=s(\operatorname{deg} h-1)+t k q+1 .
$$

What is left is to show that we can find $m, k \in Z$, and $s, t \in N$ such that

$$
s(\operatorname{deg} h-1)+t k q=-1 \text {. }
$$

Put $t=1$. It is sufficient to find $s \in N, k \in Z$, such that

$$
s(\operatorname{deg} h-1)+k q=-1 .
$$

This will be proved by showing that $((\operatorname{deg} h-1), q)=1$ and $\operatorname{deg} h-1>1$ for some $m$. But

$$
\operatorname{deg} h-1=(m p+1)^{q}-1=m^{q} p^{q}+\left(\begin{array}{l}
q \\
1
\end{array}\right) m^{q-1} p^{q-1}+\cdots+q \cdot m p .
$$


Let $m$ be any prime number greater than $p$ and $q$. Then $q \mid\left(\begin{array}{l}q \\ 1\end{array}\right)$ and $q \times m^{q} p^{q}$, which shows that $((\operatorname{deg} h-1), q)=1$ and $\operatorname{deg} h-1>q$.

We have to add that in our construction we get a representation with fixed point set of $G$ equal to $\{0\}$, as follows from the face with $(V \oplus W)^{G}=V^{G} \oplus W^{G}$ and 2.4. This completes the proof of Proposition 2.3.

The task is now to prove Theorem 2.1 for groups of even order.

2.7. Proposition. Suppose that $G$ is a finite group of even order and an odd prime divides the order of $G$.

Then there exist an orthogonal representation $V$ of $G$ with $V^{G}=$ $\{0\}$ and a G-equivariant map $f: S(V) \rightarrow S(V)$ of degree zero.

Proof. The proof is similar to that of Proposition 2.3. Let $G_{(p)}$ be the Sylow $p$-subgroup of $G, p \| G \mid$, and $G_{(2)}$ the Sylow 2-subgroup of $G$. Just as in the proof of Lemma 3, we can find an orthogonal representation $\widetilde{V}$ of $G_{(p)}$ with $\widetilde{V}_{(p)}^{G_{(p)}}=\{0\}$ and a $G_{(p)}$-equivariant map $f: S(\tilde{V}) \rightarrow S(\tilde{V})$ of degree $m p+1$, for every $m \in Z$. Also, for every $k \in Z$ we can find an orthogonal representation $\widetilde{W}$ of $G_{(2)}$ with $\widetilde{W}^{G_{(2)}}=\{0\}$ and a $G_{(2)}$-equivariant map $h: S(\widetilde{W}) \rightarrow S(\widetilde{W})$ of degree $s k+1$.

Taking the induced representations $V$-ind $\widetilde{V}, W=$ ind $\widetilde{W}$, we get $G$-equivariant maps

$$
f: S(V) \rightarrow S(V), h: S(W) \rightarrow S(W)
$$

of degrees $(m p+1)^{a}$ and $(2 k+1)^{b}$ respectively, where $a=\left|G / G_{(p)}\right|$, $b=\left|G / G_{(2)}\right|$. Note that $b$ is an odd number.

As in Proposition 2.3, we form the G-equivariant map $\stackrel{t}{1}_{1} f \stackrel{s}{L}_{1} h$ of the sphere

$$
\underbrace{S(V) * \cdots * S(V)}_{t} * \underbrace{S(W) * \cdots * S(W)}_{s}=S(t V \oplus s W) .
$$

By (2.5),

$$
\operatorname{deg}(\stackrel{t}{a} f \square \stackrel{s}{\square} h)=t(\operatorname{deg} f-1)+s(\operatorname{deg} h-1)+1 .
$$

Write $\operatorname{deg} f-1=\gamma, \operatorname{deg} h-1=\delta$.

Then $\operatorname{deg}\left(\underset{\square}{t} f \square \stackrel{s}{\square_{1}} h\right)=t \gamma+s \delta+1$. We thus have to determine $m, k \in Z$ for which $(\gamma, \delta)=1$ and $\delta<0$. 
Put $m=1$; then $\gamma=(p+1)^{a}-1$ is odd, and consequently $\gamma=$ $p_{1}^{\alpha_{1}} \cdot p_{M}^{\alpha_{M}}$, where $p_{i} \neq 2$. Take $k$ such that $2 k+1=p_{1} \cdot p_{2} \cdots p_{M}$.

Then $\delta=(2 k+1)^{b}-1=-\left(p_{1} \cdots p_{M}\right)^{b}-1<0$, and $(\gamma, \delta)=1$, since $p_{i} \mid \delta$ for every $p_{i} \in\left\{p_{1}, p_{2}, \ldots, p_{M}\right\}$. This shows that there exist $t, s \in N$ such that $t \gamma+s \delta=-1$, which proves Proposition 2.7. The proof of Theorem 2.1 is complete.

We emphasize that the proof gives more, namely the representation $V$ in the statement of Theorem 5 can be taken complex unitary.

The remainder of this section will be devoted to the proof the following statement.

2.8. THEOREM. Let $G$ be a finite p-group with at least one element of the order $p^{2}$, p-prime. Then there exist a pair of orthogonal representations $V, W$ of $G$ with $\operatorname{dim} W=\operatorname{dim} V, W^{G}=V^{G}=\{0\}$, and a G-equivariant map $f: S(V) \rightarrow S(W)$ of degree 0 .

We start with the observation that it is sufficient to consider the case $G=Z_{p^{2}}$, as it follows from 1.4. In order to prove Theorem 2.8 for $G=Z_{p^{2}}$, we need some further notation. We shall denote by $V^{1}$ the unitary representation of $Z_{p^{2}}$ of complex dimension one given by the inclusion $Z_{p^{2}} \subset S^{1}=U(1)$. Accordingly, $V^{p}$ denotes the tensor product $\underbrace{V \otimes \cdots \otimes V}_{p}$ of $V$. Note that $Z_{p^{2}}$ acts freely on $S\left(V^{1}\right)$, and for every $x \in S\left(V^{p}\right)$ the isotropy group is $Z_{p} \subset Z_{p^{2}}$.

We shall have established Theorem 6 if we prove the following

2.9. Proposition. Let $V=m V^{1}, W=m V^{p}$ be unitary representations of $Z_{p^{2}}$ with $V^{1}, V^{p}$ described above.

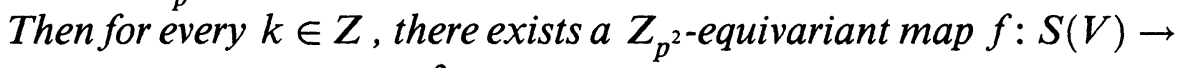
$S(W)$ of degree $p^{m}+k p^{2}$. In particular, there exists a $Z_{p^{2}}$-map $f: S(V) \rightarrow S(W)$ of degree 0 , provided $m \geq 2$.

Proof. This is a particular case of a more general result proved by C. Bowszyc ([3], Th. 3.1). In our terminology his theorem says that the function:

$$
\operatorname{deg}:[S(V), S(V)]_{Z_{p^{2}}} \rightarrow Z
$$

assigning to an equivariant homotopy class $[f]$ the degree of $f$ is a bijection onto the set $\left\{n \in Z: n=r+k p^{2}, k \in Z\right\}$ where $r$ is the degree of one particular $Z_{p^{2}}$-map $f: S(V) \rightarrow S(W)$. Taking the $Z_{p^{2}}$-map $f: S(V) \rightarrow W \backslash\{0\}$ we get, after normalization, a 
$Z_{p^{2}}$-equivariant map $f: S(V) \rightarrow S(W)$ of degree $p^{m}$. Substituting $r=p^{m}$ in Bowszyc's result we get the conclusion of Proposition 2.9. For a fuller treatment we refer the reader to [3].

Proposition 2.9 has also a very illustrative and geometric proof, the idea of which was suggested by $\mathrm{K}$. Geba. We present its main points without details. First observe that instead of a map of spheres we consider a $Z_{p^{2}}$-equivariant smooth map of pairs $f:(D(V), S(V)) \rightarrow$ $(W, W \backslash\{0\})$ a study of degree of $f$ at 0 relative $S(V)$.

Secondly, by homotopy arguments, we can assume that our $f$ is

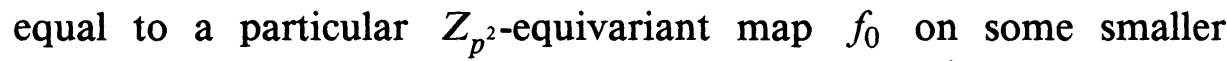
disc $D_{\zeta}(V) \subset D(V)$. It is most convenient to take $f\left(z_{1}, \ldots, z_{m}\right)=$ $\left(z_{1}^{p}, \ldots, z_{m}^{p}\right)$ as this map $f_{0}$. Since $Z_{p^{2}}$ acts freely on the ring $D(V) \backslash \stackrel{\circ}{D}_{\zeta}(V)$, by transversality arguments we can assume that zero is a regular value of $f: D(V) \backslash \stackrel{\circ}{D}_{\zeta}(V) \rightarrow W$ and $f^{-1}(0) \cap(S(V) \cup$ $\left.S_{\zeta}(V)\right)=\varnothing$. Moreover, composing $f$ if necessary with some $Z_{p^{2-}}$ diffeomorphism of $D(V) \backslash \stackrel{\circ}{\zeta}_{\zeta}(V)$ close to the identity, we can assume that there is at least one nontrivial zero of $f$ on every radius $\{t v\} \subset V, t \in R^{+}$. Having such a map, we can "kill" a particular zero orbit of $f$ composing $f$ with the $Z_{p^{2} \text {-retraction of } D(V) \text { onto }}$ $D(V)$ with some narrow conical region containing the orbit removed.

After this procedure we obtain a $Z_{p^{2}}$-equivariant map $f^{\prime}$ of degree $\operatorname{deg} f \pm p^{2}$ with the sign depending on the sign of orbit. On the other

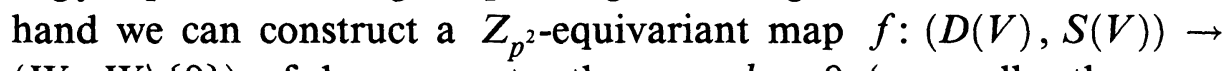
$(W, W \backslash\{0\}$ ) of degree greater than any $k>0$ (or smaller than any $k<0)$. In fact, the map

$$
f\left(z_{1}, \ldots, z_{m}\right)=\left(z_{1}^{p+k p^{2}}, z_{2}^{p}, \ldots, z_{m}^{p}\right)
$$

is $Z_{p^{2}}$-equivariant of degree $p^{m-1}\left(p+k p^{2}\right)$. Starting from such a map we can get a $Z_{p^{2}}$-equivariant map of degree required in Proposition 3.7 , by the procedure described above.

With respect to Theorems 2.1 and 2.8 the proof of Theorems 1 and 2 is complete.

2.10. Remark. As a matter of fact, every 2-group not being $Z_{2}^{r}$ contains the cyclic group $Z_{4}$ which can be checked by an induction on the order of $G$. This means that a 2-group has II.A property if and only if it is the 2-torus.

2.11. Problem. Has a finite group which all elements are of the order $p, p$ an odd prime, the Borsuk-Ulam property II.A? 
It would be desirable to give a similar classification for groups with property I.B, or II.B, but we have not been able to do this. The only natural example known to the author is the Hopf fibration. The Hopf map $\mathscr{H}: S^{3} \rightarrow S^{2}$ is an $S^{3}$-equivariant map for $S^{3}$ acting on $S^{2}$ by the projection $\pi: S^{3} \rightarrow \operatorname{SO}(3)$. This shows that $S^{3}$ fails to have property II.B. Knowing that $S^{3}$ is a subgroup of every connected nonabelian simply-connected Lie group $G$, one would like to use induced representation once more. But, unfortunately, the induced representation is infinite-dimensional, since $S^{3}$ is not cofinite. Nevertheless, this example shows that every finite subgroup of $S^{3}$ acting without fixed point on $S^{2}$ fails to have property II.B. Consequently every finite supergroup of such a group does not satisfy II.B too.

Recently [15], S. Waner gave a necessary and sufficient condition for the existence of a $G$-equivariant map, $G$-finite, from $S(V)$ into $S(W)$, where $W \subseteq V$ are orthogonal representations of $G$ and $V^{G}=$ $\{0\}$. As a consequence of his result, he got that $G=Z_{p q}, p \neq q, p, q$ primes, fails to have property I.B. This allow us to conjecture that $G$ has I.A iff it has I.B.

\section{REFERENCES}

[1] A. M. Atiyah and D. C. Tall, Group representations, $\lambda$-rings, and the J-homomorphism, Topology, 8 (1969), 253-297.

[2] K. Borsuk, Drei satze uber die- $n$ dimensionale Euklidische Sphare, Fund. Math., 21 (1933), 177-190.

[3] C. Bowszyc, On the winding number and equivariant homotopy classes of maps of manifolds with some finite group actions, Fund. Math., CXV (1983), 236-247.

[4] G. Bredon, Introduction to Compact Transformation Groups, New-York, London, Academic Press, 1972.

[5] T. tom Dieck, Transformation Groups and Representation Theory, Springer Verlag, Berlin-Heidelberg-New York, 1979.

[6] E. R. Fadell, S. Y. Husseini and P. H. Rabinowitz, Borsuk-Ulam theorems for arbitrary $S^{1}$-actions and applications, Trans. Amer. Math. Soc., 274 (1982), 343-359.

[7] W. C. Hsiang and W. Y. Hsiang, Differentiable actions of compact connected classical groups I, Amer. J. Math., 89 (1967), 705-786.

[8] C. N. Lee and A. G. Wasserman, On the group $J O(G)$, Mem. Amer. Math. Soc., 159 (1975).

[9] W. Marzantowicz, On Lefschetz numbers of a map commuting with an action of finite group, (in Polish) Ph. D. Thesis, Math. Inst. of PAS, Warsaw, 1977.

[10] - On the nonlinear elliptic equations with symmetry, J. Math. Anal. and Appl., 81, No. 1 (1981), 156-181.

[11] _ A Borsuk-Ulam theorem for an orthogonal action of the torus $T^{k}$ and the p-torus $Z_{p}^{r}$ and applications, J. Math. Anal. Appl., No. 1, (January 1989), 99-121. 
[12] L. Nirenberg, A comment on non linear problems, Proc. Conf. Catania 1981.

[13] P. Traczyk, On the G-homotopy equivalence of spheres of representations (in Polish), MSc. thesis, Dept. of Math., University of Warsaw, 1977.

[14] _ On the G-homotopy equivalence of spheres of representations, Math. Zeit., 161 (1978), 257-261.

[15] S. Waner, A note on the existence of G-maps between spheres, Proc. Amer. Math. Soc., 99 (1987), 179-181.

Received July 6, 1987. The work has been completed at the Research Center on Mathematics in Barcelona. The author wishes to express his thanks to professor Manuel Castellet for the invitation and hospitality..

UNIVERSITY OF GDAŃSK

UL. Wita Stowosza 57

80-952 Gdańsk, Poland 



\section{PACIFIC JOURNAL OF MATHEMATICS EDITORS}

\author{
V. S. VARADARAJAN \\ (Managing Editor) \\ University of California \\ Los Angeles, CA 90024-1555-05 \\ Herbert Clemens \\ University of Utah \\ Salt Lake City, UT 84112 \\ THOMAS ENRIGHT \\ University of California, San Diego \\ La Jolla, CA 92093
}

R. FINN

Stanford University

Stanford, CA 94305

Hermann Flaschka

University of Arizona

Tucson, AZ 85721

VAUGHAN F. R. Jones

University of California

Berkeley, CA 94720

Steven Kerckhoff

Stanford University

Stanford, CA 94305

\author{
C. C. MOORE \\ University of California \\ Berkeley, CA 94720
}

MARTIN SCHARLEMANN

University of California

Santa Barbara, CA 93106

HAROLd STARK

University of California, San Diego

La Jolla, CA 92093

\section{ASSOCIATE EDITORS}
R. ARENS
E. F. BECKENBACH
B. H. NeUmanN
F. WoLF
(1904-1989)
K. YosHIDA
(1906-1982)

\section{SUPPORTING INSTITUTIONS}

UNIVERSITY OF ARIZONA
UNIVERSITY OF BRITISH COLUMBIA
CALIFORNIA INSTITUTE OF TECHNOLOGY
UNIVERSITY OF CALIFORNIA
MONTANA STATE UNIVERSITY
UNIVERSITY OF NEVADA, RENO
NEW MEXICO STATE UNIVERSITY
OREGON STATE UNIVERSITY

UNIVERSITY OF ARIZONA

CAIVERSITY OF BRITISH COLUN UNIVERSITY OF CALIFORNIA

MONTANA STATE UNIVERSITY OREGON STATE UNIVERSITY

\author{
UNIVERSITY OF OREGON \\ UNIVERSITY OF SOUTHERN CALIFORNIA \\ STANFORD UNIVERSITY \\ UNIVERSITY OF HAWAII \\ UNIVERSITY OF TOKYO \\ UNIVERSITY OF UTAH \\ WASHINGTON STATE UNIVERSITY \\ UNIVERSITY OF WASHINGTON
}

The Supporting Institutions listed above contribute to the cost of publication of this Journal, but they are not owners or publishers and have no responsibility for its content or policies.

Mathematical papers intended for publication in the Pacific Journal of Mathematics should be in typed form or offset-reproduced (not dittoed), double spaced with large margins. Please do not use built up fractions in the text of the manuscript. However, you may use them in the displayed equations. Underline Greek letters in red, German in green, and script in blue. The first paragraph must be capable of being used separately as a synopsis of the entire paper. In particular it should contain no bibliographic references. Please propose a heading for the odd numbered pages of less than 35 characters. Manuscripts, in triplicate, may be sent to any one of the editors. Please classify according to the 1980 Mathematics Subject Classification (1985 Revision) scheme which can be found in the December index volumes of Mathematical Reviews. Supply name and address of author to whom proofs should be sent. All other communications should be addressed to the managing editor, or Elaine Barth, University of California, Los Angeles, California 90024-1555-05.

There are page-charges associated with articles appearing in the Pacific Journal of Mathematics. These charges are expected to be paid by the author's University, Government Agency or Company. If the author or authors do not have access to such Institutional support these charges are waived. Single authors will receive 50 free reprints; joint authors will receive a total of 100 free reprints. Additional copies may be obtained at cost in multiples of 50 .

The Pacific Journal of Mathematics (ISSN 0030-8730) is published monthly. Regular subscription rate: $\$ 190.00$ a year (12 issues). Special rate: $\$ 95.00$ a year to individual members of supporting institutions.

Subscriptions, orders for numbers issued in the last three calendar years, and changes of address should be sent to Pacific Journal of Mathematics, P.O. Box 969, Carmel Valley, CA 93924, U.S.A. Old back numbers obtainable from Kraus Periodicals Co., Route 100, Millwood, NY 10546.

The Pacific Journal of Mathematics at P.O. Box 969, Carmel Valley, CA 93924 (ISSN 0030-8730) is published monthly. Second-class postage paid at Carmel Valley, California 93924, and additional mailing offices. Postmaster: send address changes to Pacific Journal of Mathematics, P.O. Box 969, Carmel Valley, CA 93924.

\section{PUBLISHED BY PACIFIC JOURNAL OF MATHEMATICS, A NON-PROFIT CORPORATION}




\section{Pacific Journal of Mathematics}

Vol. 144, No. $2 \quad$ June, 1990

George E. Andrews and David M. Jackson, An algebraically derived $q$-analogue of a character sum associated with a class of semiregular

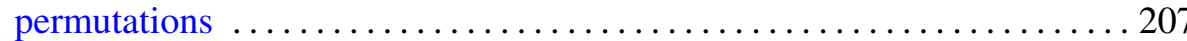

Fabio Bardelli and Andrea Del Centina, The moduli space of genus four double covers of elliptic curves is rational $\ldots \ldots \ldots \ldots \ldots \ldots \ldots \ldots 219$

Young Do Chai, An estimate of the volume of a compact set in terms of its

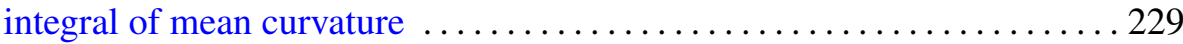

Salvador Comalada, Elliptic curves with trivial conductor over quadratic fields

Kahar El-Hussein, Résolubilité semi-globale des opérateurs différentiels invariants sur les groupes de déplacements $\ldots \ldots \ldots \ldots \ldots \ldots \ldots \ldots . \ldots 259$

David M. Goldschmidt, Classical link invariants and the Burau representation

Liliana Janicka, Radon-Nikodým problem for the variation of a vector measure

Wacław Marzantowicz, An almost classification of compact Lie groups with Borsuk-Ulam properties ............................ 299

Akira Ohbuchi, On the projective normality of some varieties of degree 5 . . 313 Ken'ichi Ohshika, Minimal measured laminations in geometric

3-manifolds

Hal Leslie Smith, A discrete Lyapunov function for a class of linear differential equations

John Samuel Spielberg, Diagonal states on $O_{2} \ldots \ldots \ldots \ldots \ldots \ldots \ldots \ldots$

Thomas Vogel, A note on the sessile drop

Gerold Wagner, On means of distances on the surface of a sphere (lower bounds) 